\title{
Quantitative Analysis of Semicrystalline Blends SAXS Data: Theoretical Modeling versus Linear Correlation Function
}

\author{
Mohamed Fatnassi, ${ }^{1}$ Fadhel Ben Cheikh Larbi, ${ }^{1}$ and Jean Louis Halary ${ }^{2}$ \\ ${ }^{1}$ Laboratoire de Physico-chimie des Matériaux Polymères, Institut Préparatoire aux Etudes Scientifiques et Techniques, BP51, \\ 2070 La Marsa, Tunisia \\ ${ }^{2}$ Laboratoire de Physico-chimie des Polymères et Milieux Dispersés (UMR 7615), Ecole Supérieure de Physique et Chimie \\ Industrielles de la Ville de Paris, 10, rue Vauquelin, 75231 Paris cedex 05, France \\ Correspondence should be addressed to Mohamed Fatnassi, mohamed.fatnassi@ipest.rnu.tn
}

Received 24 May 2010; Accepted 10 August 2010

Academic Editor: Saad A. Khan

Copyright ( $) 2010$ Mohamed Fatnassi et al. This is an open access article distributed under the Creative Commons Attribution License, which permits unrestricted use, distribution, and reproduction in any medium, provided the original work is properly cited.

\begin{abstract}
This paper compares the results yielded by two methods of small-angle X-ray scattering data analysis for semicrystalline polymer blends. The first method is based on the use of a theoretical modeling for isotropic samples and a subsequent curve fitting. The second one is a more familiar method, based on the calculation of the linear one-dimensional correlation function. The experimental material considered for this purpose deals with a series of semi-crystalline blends of poly(vinylidene fluoride) and poly(methyl methacrylate), with a PVDF content covering the range $50 \mathrm{wt} \%-100 \mathrm{wt} \%$. The results obtained by both calculation methods are systematically confronted to the crystallinity degrees deduced from wide angle X-ray scattering patterns.
\end{abstract}

\section{Introduction}

A structural periodicity was recognized in semi-crystalline polymers and polymer blends which typically consist of crystalline and amorphous domains with dimensions in the nanometer range, which form lamellar stacks embedded in a spherulitic superstructure [1]. The final properties of such a polymer or a polymer blend depend on the volume fraction of the crystalline domains and subsequently on the linear degree of crystallinity $\chi$ as well as on their size and structure. An additional important factor is the linkage which exists between the crystalline lamellae and the amorphous interlamellar regions.

In most cases, small-angle X-ray scattering (SAXS) patterns of isotropic semi-crystalline polymers and polymer blends are analyzed using the related linear one-dimensional correlation function $\gamma(r)(\mathrm{CF})$ obtained by Fourier transformation of the Lorentz-corrected experimental (SAXS) intensity distribution versus the scattering vector. Knowing $\gamma(r)$ and using the methods derived by Vonk and Kortleve $[2,3]$, and Strobl et al. (1980) $[4,5]$, one can determine the fundamental parameters of the lamellar stacks, namely, the thickness $l_{c}$ and $l_{a}$ of the crystalline lamellae and amorphous layers, respectively. The average long period $L_{p}$ can simply deduced by $L_{p}=l_{c}+l_{a}$. These quantities can also be determined by modeling the lamellar stacks structure and obtaining the best fit of a theoretical SAXS intensity distribution, calculated for the assumed model of stacks, to the experimental SAXS curve. The number of optimized parameters of the stacks depends on the complexity of the model. Generally, in the curve-fitting method, lamellar stacks are characterized by the average thickness of the crystalline lamellae $l_{c}$ and the amorphous layers $l_{a}$ and by the independent distribution functions of the crystalline lamellae and the amorphous layer thicknesses $h_{c}(z)$ and $h_{a}(z)$, respectively.

In this paper, both methods of analysis were employed and the results obtained were analyzed and compared. The measurements were performed for some semi-crystalline blends of poly(vinylidene fluoride) (PVDF) and poly(methyl methacrylate) (PMMA) over the composition of 50$100 \mathrm{wt} \%$ PVDF. It is well known that such samples are characterized by the partial crystallization of PVDF chains and by the miscibility of PMMA and residual PVDF within 
the amorphous phase. Crystallinity at equilibrium is known to increase progressively with increasing PVDF amount from 28 to $56 \mathrm{wt} \%[6,7]$. Miscibility of the amorphous phase components results from attractive interactions between PMMA carbonyl groups and atoms of PVDF [8]. In addition, two types of amorphous chains deserve to be distinguished: those which are quite far away from crystalline PVDF domains (interspherulitic amorphous) and those which are embedded within the spherulites (intraspherulitic amorphous). The interest for PVDF/PMMA blends is reinforced by the very progressive changes of crystallinity features with increasing PVDF amount.

\section{Experimental}

2.1. Materials. The PMMA used in this study was an amorphous polymer of weight average molecular weight $M_{w}=119 \mathrm{~kg} \cdot \mathrm{mol}^{-1}$. Its glass transition temperature, as determined by DSC at a heating rate of $10 \mathrm{~K} / \mathrm{min}$, was $110^{\circ} \mathrm{C}$. The grade of PVDF retained for this study presented a melt viscosity of $830 \mathrm{~Pa} \cdot \mathrm{s}$ at $230^{\circ} \mathrm{C}$, a glass transition temperature of $-40^{\circ} \mathrm{C}$, a melting temperature of $165-172^{\circ} \mathrm{C}$, and a maximum degree of crystallinity of about $50 \%$. Both polymers were kindly provided by ARKEMA.

2.2. Blending Procedure. The PVDF/PMMA samples, with, respectively, 50, 60, 70, 80, 90, and 100 weight \% of PVDF were prepared by mechanical blending using the Rheocord RC300p and the Rheomix 600p mixor systems (Polylab systems). Optimized blending conditions were set as speed of the mixor system of $40 \mathrm{rpm}$, temperature of $200^{\circ} \mathrm{C}$, and blending time of $100 \mathrm{~min}$.

2.3. SAXS Experiments. Small-angle X-ray scattering (SAXS) experiments were carried out at the "Ecole des Mines de Nancy" with $\mathrm{Cu} \mathrm{K} \alpha$ radiation, and a $3 \mathrm{~kW}$ X-ray source (Inel XRG 3000). The radiation was Ni-filtered, monochromaticized, and point collimated with a beam diameter of $\sim 1.0 \mathrm{~mm}$. With respect to the distance of $820 \mathrm{~mm}$ between sample and detector and a laminated sample thickness of $\sim 800 \mu \mathrm{m}$, the irradiation time was fixed at $2 \mathrm{~h}$. SAXS intensities were collected in a single direction, and exported to Maple and Matlab for further analysis.

\section{SAXS Data Analysis}

The procedures used to derive the structural parameters of the semi-crystalline samples under investigation have been detailed in a previous publication [9]. In fact, these methods were based on the classical analysis of the linear correlation function and the recent treatment proposed by Hsiao et al. $[10,11]$.

The one-dimensional (linear) electron correlation function, $\gamma(r)(\mathrm{CF})$, is calculated from two-dimensional scattering patterns, by choosing one direction which goes through the origin (the center of the 2D-SAXS pattern). The experimental scattering intensities are then obtained in the reciprocal space.
Prior to the calculation of the linear correlation functions, the experimental curves were smoothed using a moving average filtering $[9,10]$ and extrapolated to low and high $q$ values. At high angles, that is, at the tail of the curve, the experimental curve was fitted with a function written as a superposition of positive and negative deviations from Porod's law [12-14], this function is given by Ruland [15], assuming a lamellar stack structure with a transition layer between the amorphous and crystalline regions and a sigmoidal electron-density gradient in this layer:

$$
\lim _{q \rightarrow \infty} I(q)=I_{f l}+\frac{A_{p, q}}{q^{4}} \exp \left(-\sigma_{\operatorname{In}}^{2} q^{2}\right)
$$

where $I_{f l}$ is a constant background scattering due to electrondensity fluctuations within the phases, $A_{p, q}$ is the Porod constant, and $\sigma_{\text {In }}$ is a parameter characterizing the thickness of the transition layer. The experimental curve was also extrapolated to zero angle by fitting the function $q^{2} I(q)$ by a second-order polynomial $[9,11]$ since $q^{2} I_{\mathrm{obs}} \rightarrow 0$ as $q \rightarrow 0$. These three parameters were found by graphical selection on each experimental SAXS intensity profile and use of the Levenberg-Marquandt nonlinear least-squares fitting algorithm.

The morphological parameters of the lamellar stacks in the investigated semi-crystalline blends were determinated from the linear correlation function $\gamma(r)$, calculated by the cosine transformation $[4,5]$ of the Lorentz-corrected SAXS intensity distribution:

$$
\gamma(r)=\frac{\int_{0}^{\infty} q^{2} I(q) \cos (q r) d q}{\int_{0}^{\infty} q^{2} I(q) d q}=\frac{1}{Q} \int_{0}^{+\infty} q^{2} I(q) \cos (q r) d q
$$

where $I(q)$ is the scattering SAXS intensity, $\mathbf{q}$ is the scattering vector and $q=|\mathbf{q}|=(4 \pi / \lambda) \cdot \sin (\theta)$, with $\lambda$ and $2 \theta$ being the $\mathrm{X}$-ray wavelengh and the scattering angle, respectively, and $Q$ is the so-called second moment or invariant.

Using the linear correlation function $\gamma(r)$ (CF), the long period $L_{p}$ and the average interface thickness $e_{\text {In }}$ were determined directly [2]. The linear crystallinity $\chi_{l}$ was deduced from the linear correlation function $\gamma(r)$ using the equation $[3,9,16]$

$$
\chi_{l}\left(1-\chi_{l}\right)=\frac{D_{0}}{L_{p}}
$$

where $D_{0}$ and $L_{p}$ are the average core thickness $[2,4]$ and the long period, respectively. The average core thickness $D_{0}$ is the position at the end of the linear section in the CF $\gamma(r)$.

From the two solutions of the above quadratic equation, the one with the higher value should be ascribed to the larger fraction of the two phases found within the lamellar stacks.

The thickness of the crystalline $l_{c}$ and amorphous $l_{a}$ layers in the stacks were then calculated as follows:

$$
\begin{gathered}
l_{c}=\chi_{l} L_{p}, \\
l_{a}=\left(1-\chi_{l}\right) L_{p} .
\end{gathered}
$$




\section{Theoretical Modeling}

The evaluation of the SAXS profiles was carried out referring to Hosemann model [17]. In fact, in the framework of this model, a semi-crystalline polymer blend is considered as a system of stacks of lamellar crystals separated by amorphous layers. The lamellae are assumed to be flat and of infinite lateral dimensions. All stacks are statistically identical and have the same characteristics. The fundamental parameters of such model are the average thicknesses of the crystalline lamellae $l_{c}$ and the amorphous layers $l_{a}$ and the average long period of the stack $L_{p}$. The height of the stacks is assumed to be much larger than the long period. This assumption, in practice, takes into account a monodimensional electron density change along the normal direction to the lamellae. The thickness of the crystalline lamellae and amorphous layers vary independently and are described by independent distribution functions $h_{c}(z)$ and $h_{a}(z)$. The theoretical SAXS intensity of such a one-dimensional system in the direction perpendicular to the lamellae is the sum of two components:

$$
J_{1}(q)=J_{B}(q)+J_{C}(q),
$$

where $J_{B}(q)$ is determined by the electron-density distribution in a stack and $J_{C}(q)$ represents the scattering intensity of a whole stack as an isolated object. According to Hosemann and Bagchi [17],

$$
\begin{gathered}
J_{B}(q)=\frac{\Delta \rho^{2}}{q^{2} L_{p}} \operatorname{Re}\left[\frac{\left(1-f_{c}\right)\left(1-f_{a}\right)}{\left(1-f_{c} f_{a}\right)}\right], \\
J_{C}(q)=\frac{2 \Delta \rho^{2}}{q^{2} N L_{p}} \operatorname{Re}\left[\frac{f_{a}\left(1-f_{c}\right)^{2}\left(1-\left(f_{a} f_{c}\right)^{N}\right)}{\left(1-f_{c} f_{a}\right)^{2}}\right] .
\end{gathered}
$$

Re means the real part of the quantity under brackets. $\Delta \rho$ is the difference in electron density between crystalline and amorphous phases, $N$ is the number of lamellae, in a stack, and $f_{c}$ and $f_{a}$ are the Fourier transforms of the distribution functions $h_{c}(z)$ and $h_{a}(z)$ respectively. When the height of stacks is large compared to the X-ray wavelength $\left(N L_{p} \gg \lambda\right)$, that is, for stacks containing a sufficiently large number of lamellae the intensity term $J_{C}(q)$ can be ignored in the experimentally accessible region. Assuming that the thickness distributions $h_{c}(z)$ and $h_{a}(z)$ are described by Gaussian functions, the corresponding Fourier transforms are given by

$$
\begin{aligned}
& f_{c}(q)=\exp \left(i \ell_{c} q\right) \exp \left(\frac{-\sigma_{c}^{2} q^{2}}{2}\right), \\
& f_{a}(q)=\exp \left(i \ell_{a} q\right) \exp \left(\frac{-\sigma_{a}^{2} q^{2}}{2}\right),
\end{aligned}
$$

where $\sigma_{c}$ and $\sigma_{a}$ are the standard deviations of $h_{c}(z)$ and $h_{a}(z)$ distributions.

Knowing that the scattering intensity $I_{\text {mes }}(q)$ measured from an isotropic system can be transformed into the onedimensional intensity $J_{1}(q)$ by Lorentz correction $[18,19]$

$$
J_{1}(q)=c I_{\text {mes }}(q) q^{2},
$$

where $\mathrm{c}$ is a proportionality constant.
The final formula for the theoretical SAXS intensity profile $I_{1}(q)$ used in this work is given by $[15,17,20,21]$

$$
I_{1}(q)=\frac{I_{1,0}}{q^{4}} \exp \left(-\sigma_{\operatorname{In}}^{2} q^{2}\right) \operatorname{Re}\left[\frac{\left(1-f_{c}\right)\left(1-f_{a}\right)}{\left(1-f_{c} f_{a}\right)}\right],
$$

where $I_{1,0}$ is a constant and the introduced exponential term in the numerator accounts for the changes in the SAXS intensity profile caused by a transition layer between crystalline and amorphous regions [10] and $\sigma_{\text {In }}$ is a parameter characterizing a sigmoidal electron-density profile in this layer.

In fact, $H(q)=\exp \left(-1 / 2 \sigma_{\operatorname{In}}{ }^{2} q^{2}\right)$ represents the Fourier transform of the interfacial smoothing electron-density function [20] which is assumed to be a Gaussian function.

The parameters of the employed model were found by best fit of the theoretical SAXS intensity profile $I_{1}(q)$ to the experimental SAXS intensity $I(q)$.

Maple software worksheet was used to write implicitly and to compute formally (explicitly) the theoretical expression of the SAXS intensity $I_{1}(q)$. Again, maple "PlotAnimate" tool was used to investigate the effects of the different parameters values on the theoretical SAXS intensity profile $I_{1}(q)$. Furthermore, this tool can provide an objective comparison between measured $I(q)$ and theoretical $I_{1}(q)$ SAXS intensity profiles, it permits again to look for physically acceptable starting values of the independent parameters before carrying out the curve-fitting tasks.

Finally, a compatible form of the theoretical expression of the SAXS intensity $I_{1}(q)$ was exported to Matlab software for curve-fitting procedures.

In each curve-fitting session, six independent and decoupled parameters were optimized according to the best least-squares fit, using the classical Levenberg-Marquandt nonlinear least-squares fitting algorithm with the robust trust region method: the average thickness of the crystalline lamellae $l_{c}$ and its standard deviation $\sigma_{c}$, the average thickness of the amorphous layers $l_{a}$ and its distribution standard deviation $\sigma_{a}$, the transition layer parameter $\sigma_{\text {In }}$, and the scaling factor $I_{1,0}$. Before the fitting, the scaling factor was estimated and a constant background was determinated from experimental curve $I(q)$ using the method described above in the paragraph dedicated to SAXS data analysis.

From this direct curve-fitting method, the resulting linear crystallinity $\chi(\%)$ value is simply obtained by

$$
\chi(\%)=\frac{l_{c}}{L_{p}}=\frac{l_{c}}{\left(l_{c}+l_{a}\right)} .
$$

\section{Results and Discussion}

The theoretical SAXS profiles were fitted to the experimental ones over a broad range of scattering vectors $q$ with $99 \%$ confidence bounds for parametric fits. Whatever the sample under study may be, good fits were observed, as shown in Figures $1(\mathrm{a})-1(\mathrm{f})$ with the root mean squared error (RMSE) for each fit. 


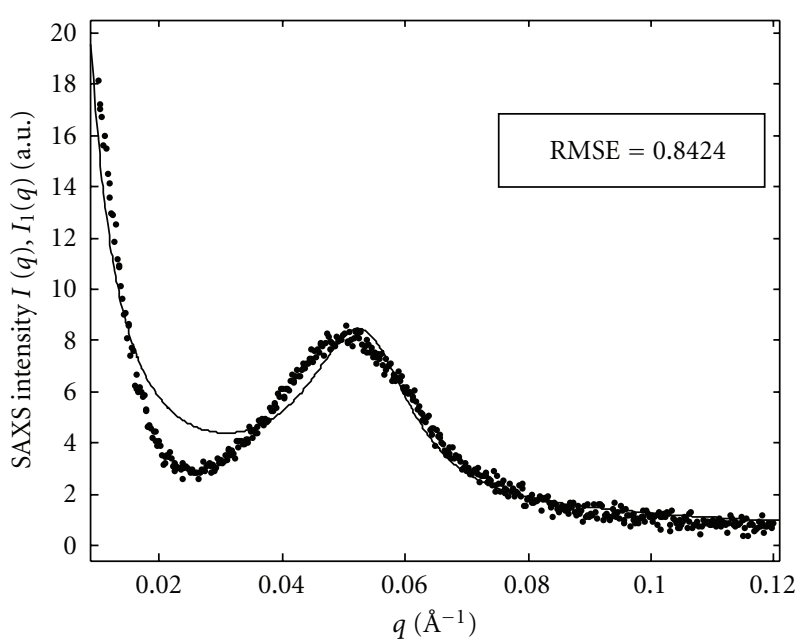

(a)

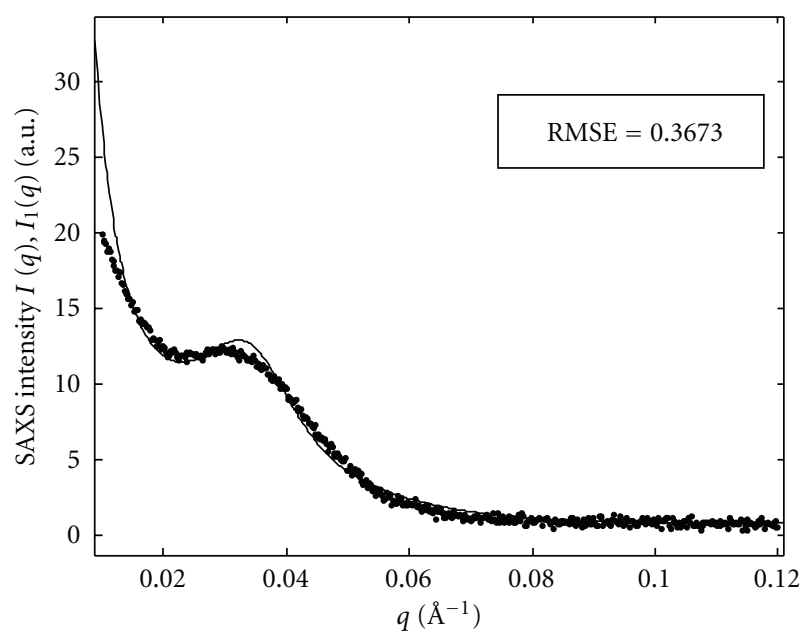

(c)

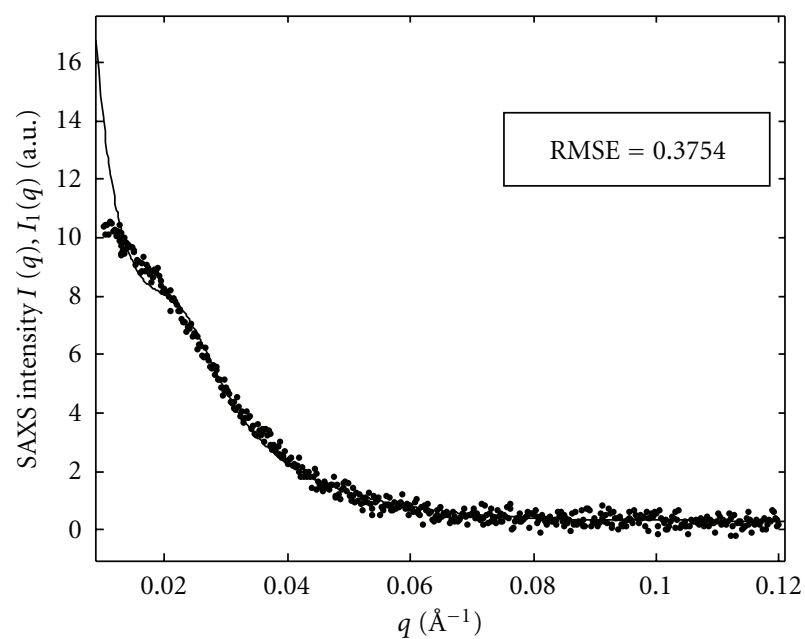

- $I(q)$

- Fit $I_{1}(q)$

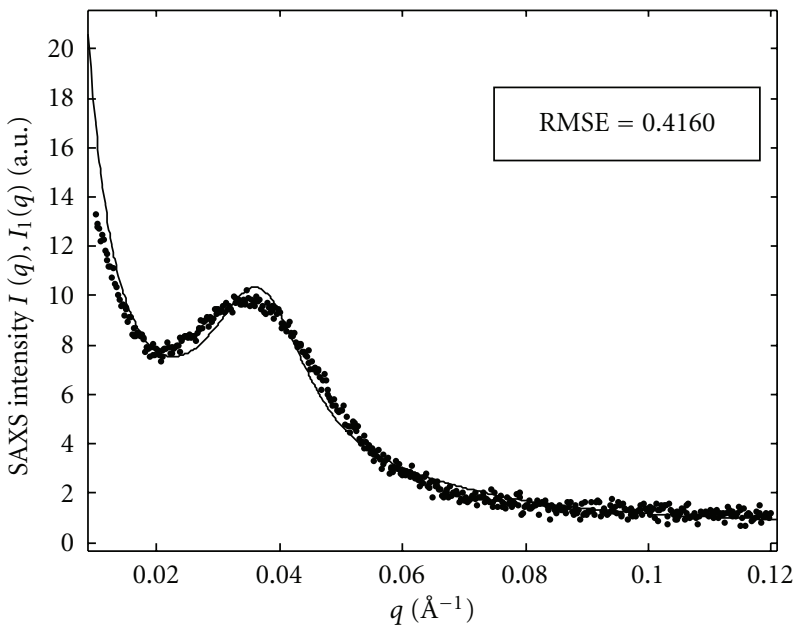

(b)

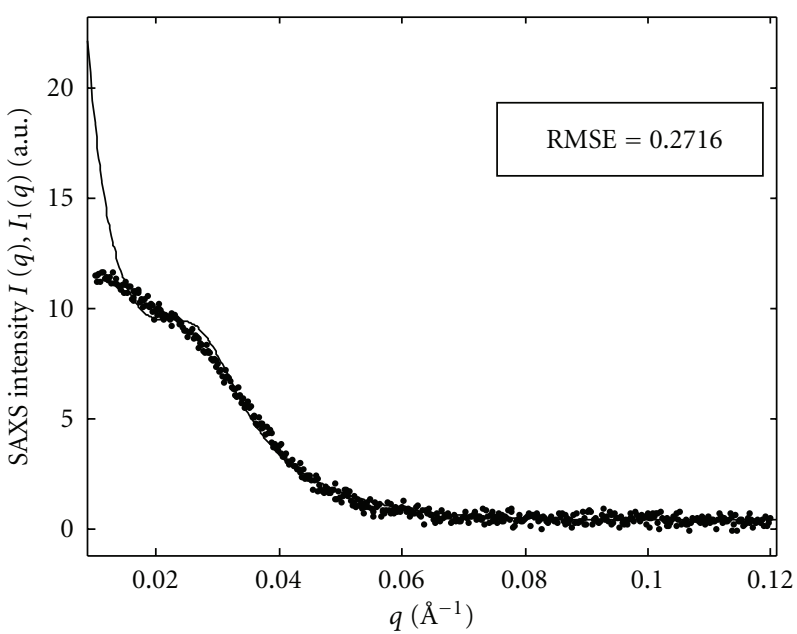

(d)

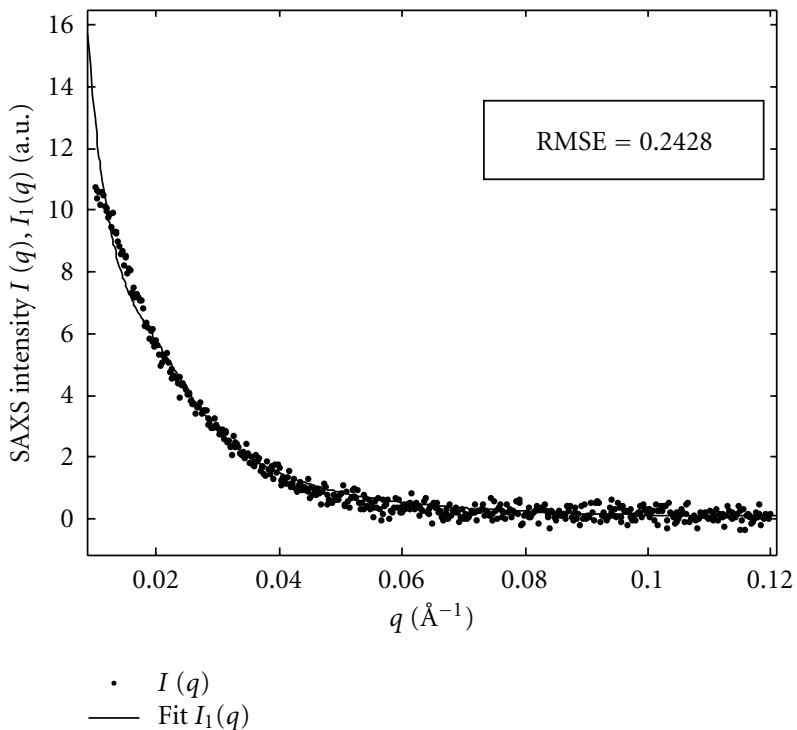

(f)

FIGURe 1: Best fit between theoretical $I_{1}(q)$ and experimental $I(q)$ SAXS intensity profiles for (a) pure PVDF; (b) $90 \mathrm{wt} \%$ PVDF sample; (c) $80 \mathrm{wt} \%$ PVDF sample; (d) $70 \mathrm{wt} \%$ PVDF sample; (e) $60 \mathrm{wt} \%$ PVDF sample; (f) $50 \mathrm{wt} \%$ PVDF sample. 


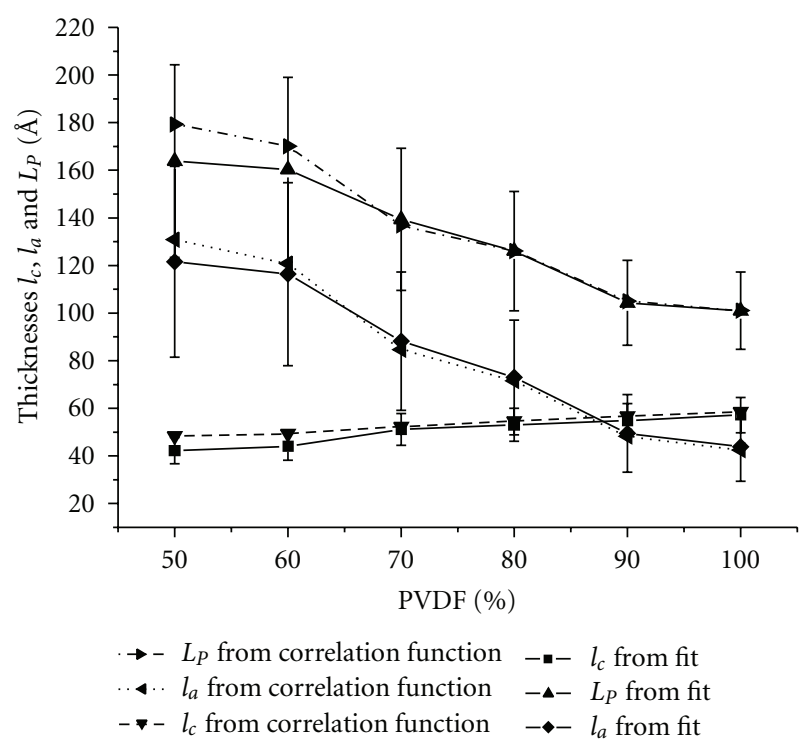

FIGURE 2: Thickness, $l_{c}$, of the crystalline lamellae, thickness of the amorphous layers, $l_{a}$ and average long period, $L_{p}$, deduced from linear correlation function $\gamma(r)$ and curve-fitting.

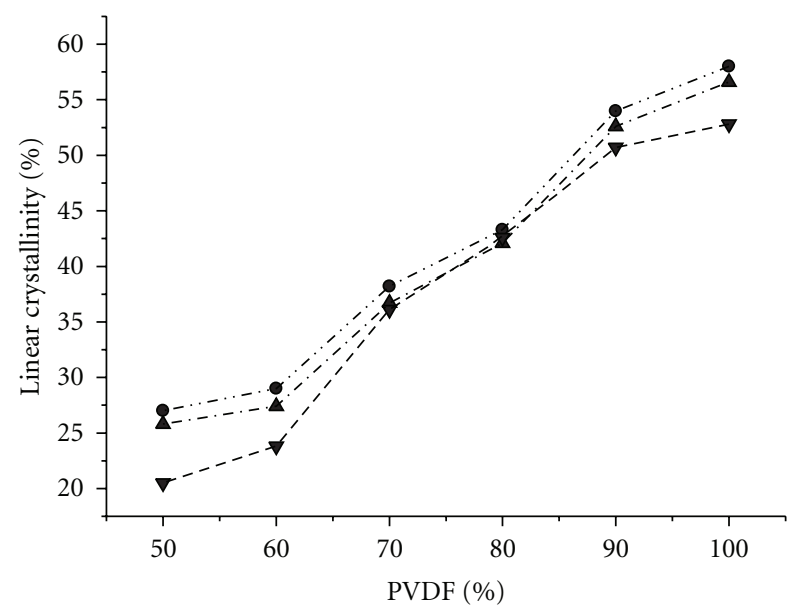

- - Crystallinity from linear correlation function

- - Crystallinity from curve-fitting

$-\nabla-$ Crystallinity from WAXS

Figure 3: Linear crystallinity values deduced from linear correlation function $\gamma(r)$ analysis, curve-fitting, and WAXS.

Moreover, for all the investigated samples, the parameters of the lamellar stacks determined on the best fits agree quite well with those obtained from the linear correlation function $\gamma(r)$. The parameters calculated using the two methods are compared in Figure 2. It turns out that the values of $L_{p}$ and $l_{a}$ determined with the curve-fitting method are about $5 \%-$ $11 \%$ lower than those determined from the linear correlation function $\gamma(r)$. This result might have been anticipated. In general, the long period calculated from the linear correlation function represents the most probable value and is relatively higher than the mean value, particularly when noticeable deviations from regular periodicity occur in the stacks

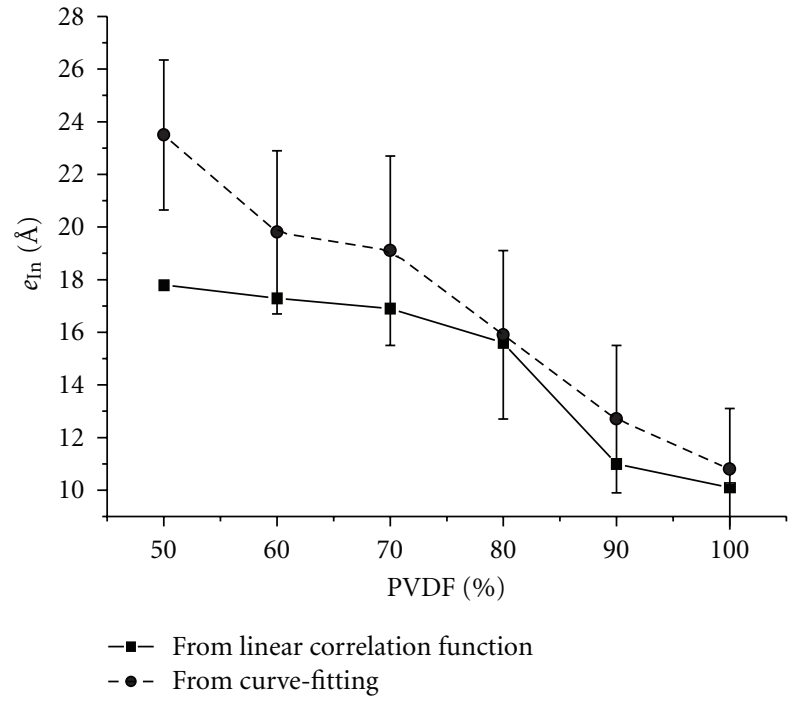

FIGURE 4: Average interface thickness deduced from linear correlation function $\gamma(r)$ and curve-fitting.

[22]. Such is the case for the samples under study here, as the reduced standard deviation $\sigma_{a} / l_{a}$ of $l_{a}$ was found to be in the range $0.23-0.55$. On the contrary, the thickness of the crystalline lamellae determined from the linear correlation function is related to the number average value [22]. This is why the differences between the values of this parameter determined from curve-fitting and from $\gamma(r)$ are less than $4 \%$.

The linear crystallinity values deduced from SAXS data, that is, from linear correlation function $\gamma(r)(\mathrm{CF})$ and from curve-fitting, are compared with the values obtained by WAXS [7]. The plots of linear crystallinity versus \%PVDF with the two methods are very close to each other and exhibit the same trends (Figure 3).

For most of the investigated samples, the values of the average interface thickness determined from curve-fitting agree very well with those obtained with the method of the linear correlation function $\gamma(r)(\mathrm{CF})$, (Figure 4). However, the samples with $50 \%$ and $60 \%$ PVDF display relatively smaller values of the linear crystallinity $\chi_{\text {WAXs }}$ and relatively higher values of the average interface thickness $e_{\text {In }}$. An intermediate behavior is observed for the 70\% PVDF sample, particularly for the average interface thickness $e_{\mathrm{In}}$.

Furthermore, and again for the two samples with 50\% and $60 \%$ PVDF, the values of $l_{a}$ obtained in the curve-fitting analysis were relatively lower than those obtained in the (CF) analysis. Interestingly, it was also found that slightly higher values of $e_{\text {In }}$ were obtained in the curve-fitting analysis compared to those obtained in the (CF) analysis. Possibly, the amorphous domains contain a part of the interface in the (CF) analysis whereas a part of these amorphous domains corresponds in reality to the interface layer or to the crystalline phase in the curve-fitting analysis.

In general, the linear crystallinity values found with SAXS method are slightly higher than those obtained from WAXS, because a part of the transition layer between the crystalline and amorphous phases is taken as crystalline in this method. 


\section{Conclusion}

The consistency of the results obtained confirms that the simple Hosemann model of lamellar stacks used in this work correctly describes the real structure of investigated PVDF/PMMA samples and can be successfully employed in the analysis of SAXS curves.

It was found that, the interface modeling incorporated into the scattering model by adding the exponential factor $H^{2}(q)$ with $H(q)$ representing the Fourier transform of the interfacial smoothing electron-density function, in the numerator of (9), in addition to producing negative deviations from Porod's law, this factor will also affect the SAXS intensity profile at the whole of the $q$-range.

We have shown in this paper that the SAXS intensity in reciprocal space can be directly fitted by a suitable model. In fact, the SAXS intensity profile calculated from the lamellar structure model based on the Hosemann theoretical network is directly and explicitly least-squares fitted to the observed SAXS intensity, and the various parameters of the model are refined. It was found that direct SAXS intensity profile analysis in reciprocal space provides a more objective and directly meaningful approach especially when the lamellar peak is clearly defined. The linear correlation function (CF) analysis provides more meaningful results even when the lamellar peak is not clearly defined.

\section{Acknowledgments}

This study was supported by the "Comite Mixte de Coopération Franco-Tunisienne" (CMCU). The PVDFPMMA SAXS spectra used in our calculations have been collected by J. Jarray, J. M. Hiver, A. Dahoun, and C. G'Sell at "Ecole des Mines de Nancy". These colleagues are warmly acknowledged for their contribution to the achievement of the present study. Thanks are also due to Dr. A. Dubault for valuable discussions and constant encouragements during the stays in France of one of us (M. Fatnassi).

\section{References}

[1] B. Wunderlich, Macromolecular Physics, Volume 1, Academic Press, New York, NY, USA, 1970.

[2] G. Kortleve and C. G. Vonk, "X-ray small-angle scattering of bulk polyethylene. III. Results," Kolloid-Zeitschrift, vol. 225, no. 2, pp. 124-131, 1968.

[3] C. G. Vonk and G. Kortleve, "X-ray small-angle scattering of bulk polyethylene. II. Analyses of the scattering curve," Kolloid-Zeitschrift, vol. 220, no. 1, pp. 19-24, 1967.

[4] G. R. Strobl and M. Schneider, "Direct evaluation of the electron density correlation function of partially crystalline polymers," Journal of Polymer Science Part A, vol. 18, no. 6, pp. 1343-1359, 1980.

[5] G. R. Strobl, M. J. Schneider, and I. G. Voight-Martin, "Model of partial crystallization and melting derived from small-angle $\mathrm{X}$-ray scattering and electron microscopic studies on lowdensity polyethylene," Journal of Polymer Science Part A, vol. 18, no. 6, pp. 1361-1381, 1980.

[6] J. Jarray, F. B. C. Larbi, F. Vanhulle, A. Dubault, and J. L. Halary, "Thermal and mechanical behavior of amorphous and semi-crystalline poly(vinylidene fluoride)/poly(methyl methacrylate) blends," Macromolecular Symposia, vol. 198, pp. 103-116, 2003.

[7] J. Jarray, Elaboration, caractérisation et étude des propriétés mécaniques de mélanges amorphes et semi-cristallins de polyfluorure de vinylidène-polyméthacrylate de méthyle, M.S. thesis, University El Manar, Tunis, Tunisia, February 2005.

[8] C. Léonard, J. L. Halary, and L. Monnerie, "Hydrogen bonding in PMMA-fluorinated polymer blends: FTi.r. investigations using ester model molecules," Polymer, vol. 26, no. 10, pp. 1507-1513, 1985.

[9] M. Fatnassi, F. Ben Cheikh Larbi, A. Dubault, and J. L. Halary, "Structural study of semi-crystalline blends of poly(vinylidene fluoride) and poly(methyl methacrylate) by means of linear correlation and interface distribution functions," E-Polymers, vol. 2005, no. 056, 2005.

[10] B. S. Hsiao and R. K. Verma, "A novel approach to extract morphological variables in crystalline polymers from timeresolved synchrotron SAXS data," Journal of Synchrotron Radiation, vol. 5, no. 1, pp. 23-29, 1998.

[11] Z.-G. Wang, B. S. Hsiao, and N. S. Murthy, "Comparison of intensity profile analysis and correlation function methods for studying the lamellar structures of semicrystalline polymers using small-angle X-ray scattering," Journal of Applied Crystallography, vol. 33, no. 3 I, pp. 690-694, 2000.

[12] G. Porod, "Die Röntgenkleinwinkelstreuung von dichtgepackten kolloiden Systemen. I. Teil," Kolloid-Zeitschrift, vol. 124, no. 2, pp. 83-114, 1951.

[13] G. Porod, "Die Röntgenkleinwinkelstreuung von dichtgepackten kolloiden Systemen. II. Teil," Kolloid-Zeitschrift, vol. 125, no. 1, pp. 51-57, 1952.

[14] G. Porod, "Die Röntgenkleinwinkelstreuung von dichtgepackten kolloiden Systemen. II. Teil," Kolloid-Zeitschrift, vol. 125, no. 2, pp. 108-122, 1952.

[15] W. Ruland, "Small-angle scattering of two-phase systems: determination and significance of systematic deviations from Porod's law," Journal of Applied Crystallography, vol. 4, pp. 7073, 1971.

[16] Z. Denchev, A. Nogales, T. A. Ezquerra, J. FernandesNascimento, and F. J. Baltà-Calleja, "On the origin of the multiple melting behavior in poly(ethylene naphthalene-2,6dicarboxylate): microstructural study as revealed by differential scanning calorimetry and X-ray scattering," Journal of Polymer Science Part B, vol. 38, no. 9, pp. 1167-1182, 2000.

[17] R. Hosemann and S. N. Bagchi, Direct Analysis of Diffraction by Matter, North Holland, Amsterdam, The Netherlands, 1962.

[18] F. J. Baltà-Calleja and G. G. Vonk, X-Ray Scattering of Synthetic Polymers, Elsevier Science, New York, NY, USA, 1989.

[19] B. Crist and N. Morosoff, "Small-angle X-ray scattering of semicrystalline polymers. II. Analysis of experimental scattering curves ," Journal of Polymer Science Part B, vol. 11, no. 6, pp. 1023-1045, 1973.

[20] J. T. Koberstein, B. Morra, and R. S. Stein, "The determination of diffuse-boundary thicknesses of polymers by small-angle Xray scattering," Journal of Applied Crystallography, vol. 13, no. 1, pp. 34-45, 1980.

[21] J. Rathje and W. Ruland, "Density fluctuations in amorphous and semicrystalline polymers," Colloid and Polymer Science, vol. 254, no. 3, pp. 358-370, 1976.

[22] B. Crist, "Analysis of amall-angle X-ray scattering patterns," Journal of Macromolecular Science Part B, vol. 39, no. 4, pp. 493-518, 2000. 

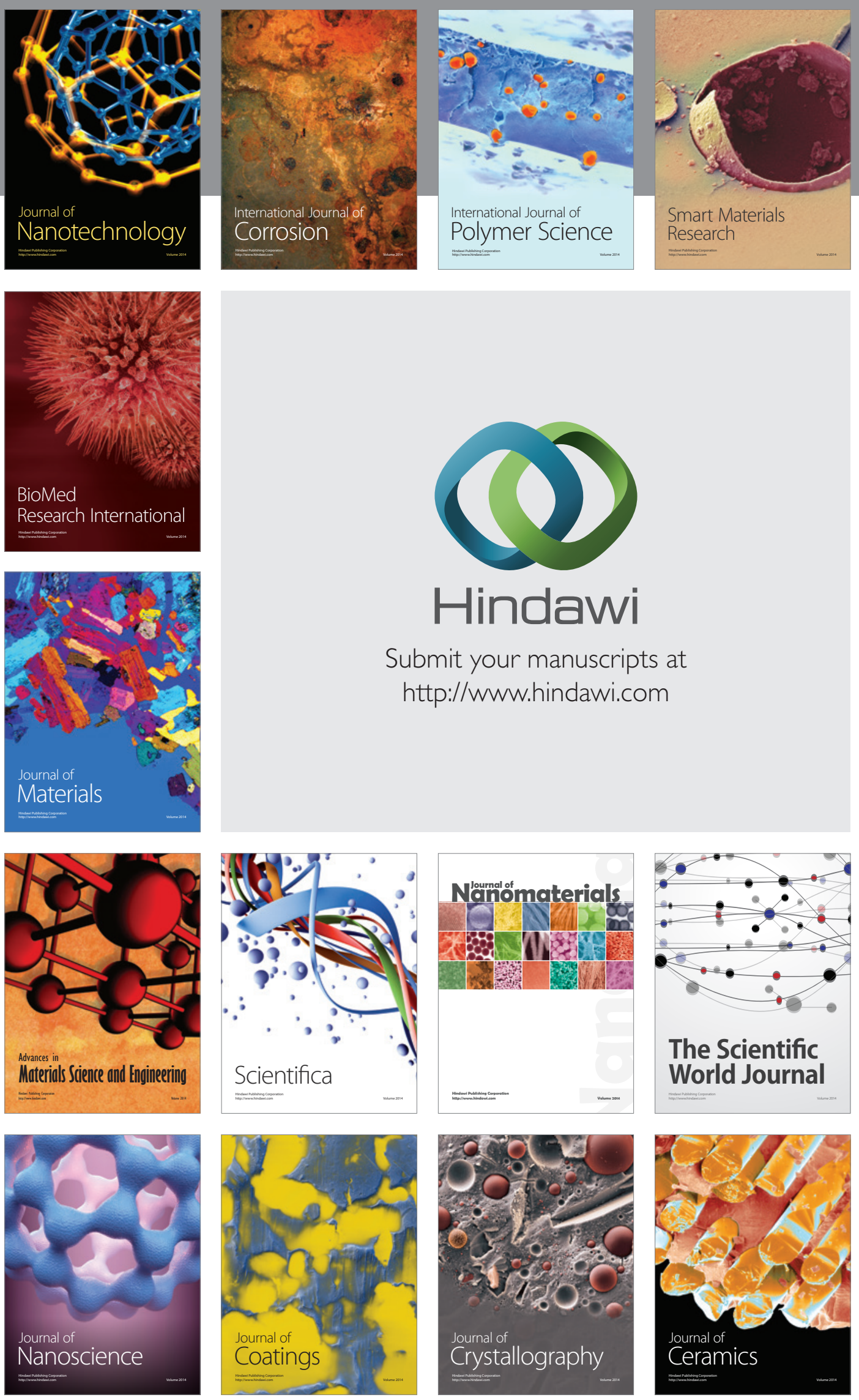

The Scientific World Journal

Submit your manuscripts at

http://www.hindawi.com

\section{World Journal}

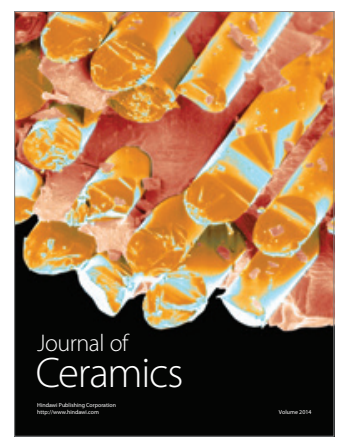

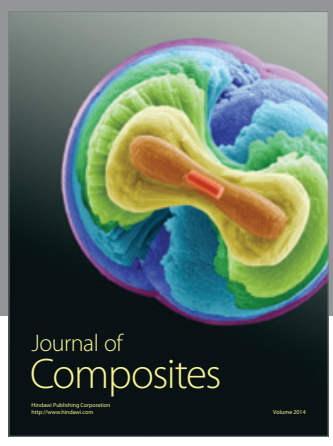
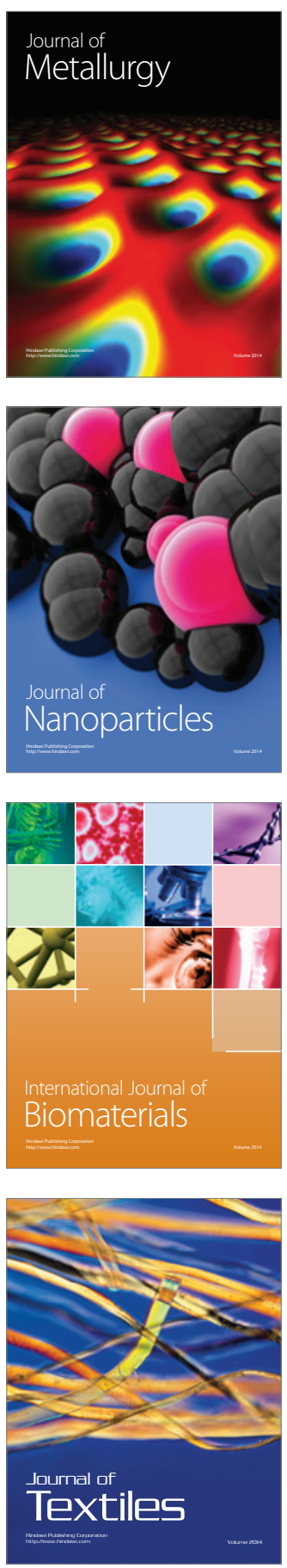